\title{
EXHIBITION
}

\section{Lighting up the background}

Intermittent flashes of light were seen emanating at night from the physics department at Queen Mary, University of London, for six weeks last year. They were triggered by background radiation: subatomic particles detected by a hundred Geiger counters connected to photography flash-bulbs.

The installation What the Eye Can't See the Heart Can't Grieve For - devised by artist Matthew Tickle, in collaboration with physicist Fay Dowker, who seeks to interpret quantum physics - contradicts the adage provided by its title. Itremindsus that the invisible can be dangerous.

The experience is now captured on DVD, where some images, such as the one shown here, evoke the surreal façades painted by René Magritte. An accompanying booklet remarks that "the work exists only as a series of moments of perception, as imprints on our retinas. The moment of

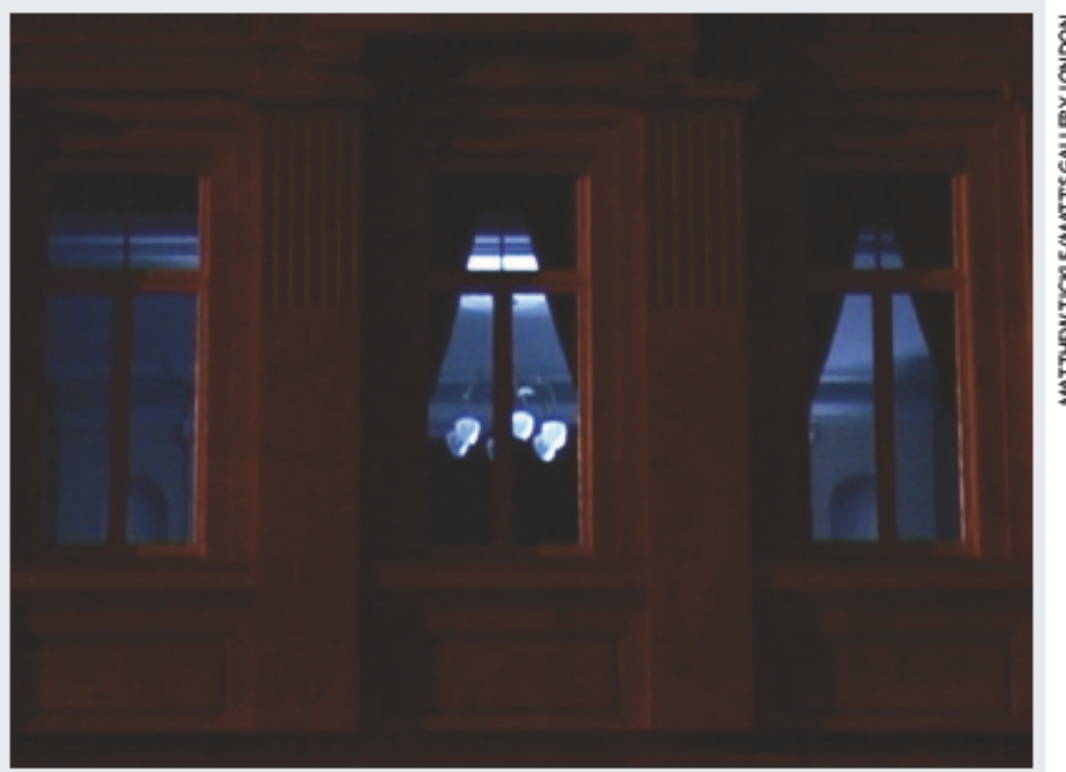

\section{A philosopher's vision}

\section{Action in Perception \\ by Alva Noë \\ MIT Press: 2005.392 pp. $\$ 38, £ 24.95$}

\section{Richard L. Gregory}

Philosophers contribute significantly to the brain sciences by clarifying terminology and concepts, occasionally issuing radical challenges and stimulating suggestions. It might be said for the whole of science that conceptual significance is as important as statistical significance, and philosophy contributes most in areas where generally accepted paradigms are lacking, such as cognitive brain science. The mystery of consciousness makes scientists listen to even the wildest ideas and most extreme challenges of philosophers. These are most effective when the philosopher has a grasp of the physiology and key experimental data.

Action in Perception by the philosopher Alva Noë is a short and clearly written book that abounds in such challenges. Like books by Daniel C. Dennett, the pioneer in this area, it discusses both phenomena and experiments. No doubt brain scientists would have liked the brain to be central to the book, but although it is mentioned occasionally, 'brain' does not even qualify for a place in the index.

Noë objects to theories of vision as 'snapshots', stressing that it is built up from many fixations of the moving eyes - rather like discovering the form of objects by exploring them by touch. Touching bits of a bottle with the eyes shut is sufficient to experience the whole bottle. If you look at the front surface of a tomato, you see the whole tomato as an object, even appreciating what is inside. Noë makes a useful distinction between 'seeing' and 'presence'.

This is really a battle with straw men, however, as comparing seeing with touching goes back to George Berkeley in the eighteenth century. The comparison was also made in the middle of the nineteenth century by Hermann von Helmholtz, the founder of the science of visual perception. Von Helmholtz stressed the importance of eye movements (which until recently were hard to record, but were not ignored), and the parallels between active touch and vision, in an eloquent account of the importance of explorative touching in helping children learn to see and understand. For followers of von Helmholtz, it is not only the extra information provided by touch that is important for seeing - knowledge of many other non-optical features, including those sensed by taste, smell and sound, contribute to making the tenuous images in the eyes useful.

Berkeley's concept of a blind man exploring spatial relations by tapping his stick is developed differently by Noë in his 'enactive theory.' This ambitious theory tries to make seeing even more closely related to the object world than touching, despite the intervening retinal image and the physiological complexity of the visual channels and brain processing. It claims that seeing colours is similar to feeling objects: for Noë, colours exist objectively in objects, so there would be colours in the world even if there were no eyes or brains. This was denied by Newton and John Locke in the seventeenth century, and by current brain science, which posits that colours are created in brain regions, although how this happens is still a mystery, as consciousness is not understood.

Philosophy and science do battle over phenomena such as not seeing a blank hole off the centre of this page, where the image falls on the 'blind spot' of the retina. Although there are no retinal signals from this large region, we do not see blackness - or nothing — but a complete page. Is the missing region actively filled in, by extrapolation from its surroundings? Or is it, as Dennett and Noë believe, passively ignored (like a boring person at a party), as it never provides useful information? This point is well worth considering, as it is easy to bark up the wrong tree, or (if you will) to bark up trees that do not exist; extensive barking may suggest the presence of non-existent trees, leading to the creation of mythologies. There are experiments that support mechanisms for active filling-in, some published in Nature. But the debate over active filling-in versus passive ignoring is a controversial issue for many perceptual phenomena, with very different implications, some of them clinically important.

Philosophers' accounts of consciousness have special interest as experiments are few and far between and are extremely hard to interpret. Perhaps the most interesting discussions in this book are centred on the radical scepticism known as the 'grand illusion', initiated by Dennett and recently discussed by others including Susan Blackmore. The challenging claim is that we are misled by our consciousness into thinking of visual experience as continuous and rich, whereas it is really discontinuous and sketchy. This view is apparently supported by striking phenomena such as change blindness and inattention blindness, which underlie much of conjuring.

Noe's balanced, well-considered account of this hot topic makes the claim - contrary to most brain scientists - that the brain does not provide an internal representation of the world. Surely Noë is right to ask how much is represented by the brain, and how much of what we seem to see is illusion? No doubt this is an empirical question, but philosophy serves a useful purpose in asking it in ways that may be answered experimentally. So philosophy and science meet, to mutual benefit.

Richard L. Gregory is in the Department of Experimental Psychology, University of Bristol, Bristol BS81TN, UK. 